\title{
Laser Beam Modeling in Optical Storage Systems
}

J.P. Treptau, Optical Sciences Center, University of Arizona. Tucson, AZ. 85721

T.D. Milster, Optical Sciences Center, University of Arizona, Tucson, AZ. 85721

D.G. Flagello, T.J. Watson Research Center, IBM, Yorktown Heights, NY, 10598

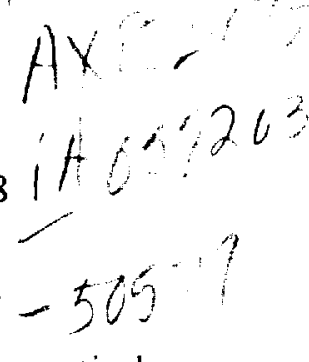

\section{Abstract}

A computer model has been developed that simulates light propagating through an optical data storage system. This paper will discuss a model of a laser beam that originates at a laser diode, propagates through an optical system, interacts with a optical disk, reflects back from the optical disk into the system, and propagates to data and servo detectors.

\section{Introduction}

The field of optical data storage technology has rapidly advanced in the recent past. Several different types of optical disks have been developed, each with different capabilities and different methods of storing information. One form of optical disk used for data storage is the CD-ROM, which is a read only device. The write-once read-many (WORM) medium was developed after the CD-ROM, and it has the increased functionality of allowing the user to write information to the disk. A drawback of WORM devices is that they are not erasable. Most recently, the erasable optical disk has been developed, which has the full functionality of magnetic disk media, in that data may be written, read back, and erased from the disk if desired. This paper will discuss a system model of an erasable optical disk system. A number of system models analyzing various components of an optical disk system have already been completed $(1)(2)(3)$. In contrast to those models, this model is a complete, end-to-end system model instead of an analysis that deals with only specific components of an optical data storage system.

There are several types of erasable optical disks, each with a different technique to write, read, and erase data. The most common type of erasable media is magneto-optic. The read operation in magneto-optic media accomplished through polarization modulation. Light incident on the disk is reflected, and the reflected light has its polarization rotated due to the data pattern on the disk. The polarization rotation of the reflected light is known as the polar Kerr effect (4). The system model discussed in this paper will consider the disk to be an erasable, magneto-optic medium.

\section{Modeling the Laser Beam}

The source of light in a magneto-optic (M-O) disk system is a laser diode. A physical optics analysis is accomplished through modeling the amplitude and phase of the laser beam's electric field. Beam propagation is simulated through the use of Fresnel diffraction calculations (5). In this model, a scalar diffraction approach is used. $\mathbf{A}$ vector diffraction analysis is certainly valid, but it is very computationally expensive. In the system model, the beam is represented by a matrix of complex values, where each matrix element represents a different sample point in the wavefront. The complex values represent the amplitude and phase of the wavefront at a given point. Fresnel diffraction calculations are computed through the use of Fast Fourier Transforms.

Consideration must be given to the type of beam a laser diode generates. Among others, there are two characteristics of a typical laser diode used for optical data storage that are discussed. A diagram for a typical laser diode is shown in Figure 1. The cavity is rectangular in shape, which causes 
the far-field pattern of the beam to have an elliptical shape. This necessitates, as far as optical data storage is concerned, circularizing optics in the system. Secondly, the cavity is index guided perpendicular to the diode junction, and gain guided parallel to the junction. This has three effects on the emitted beam: (1) The far-field intensity pattern has a Lorentzian profile in the direction perpendicular to the junction (6); (2) The farfield intensity pattern has a Gaussian profile in the direction parallel to the junction (7); and (3) the beam contains astigmatism (ㄱ).

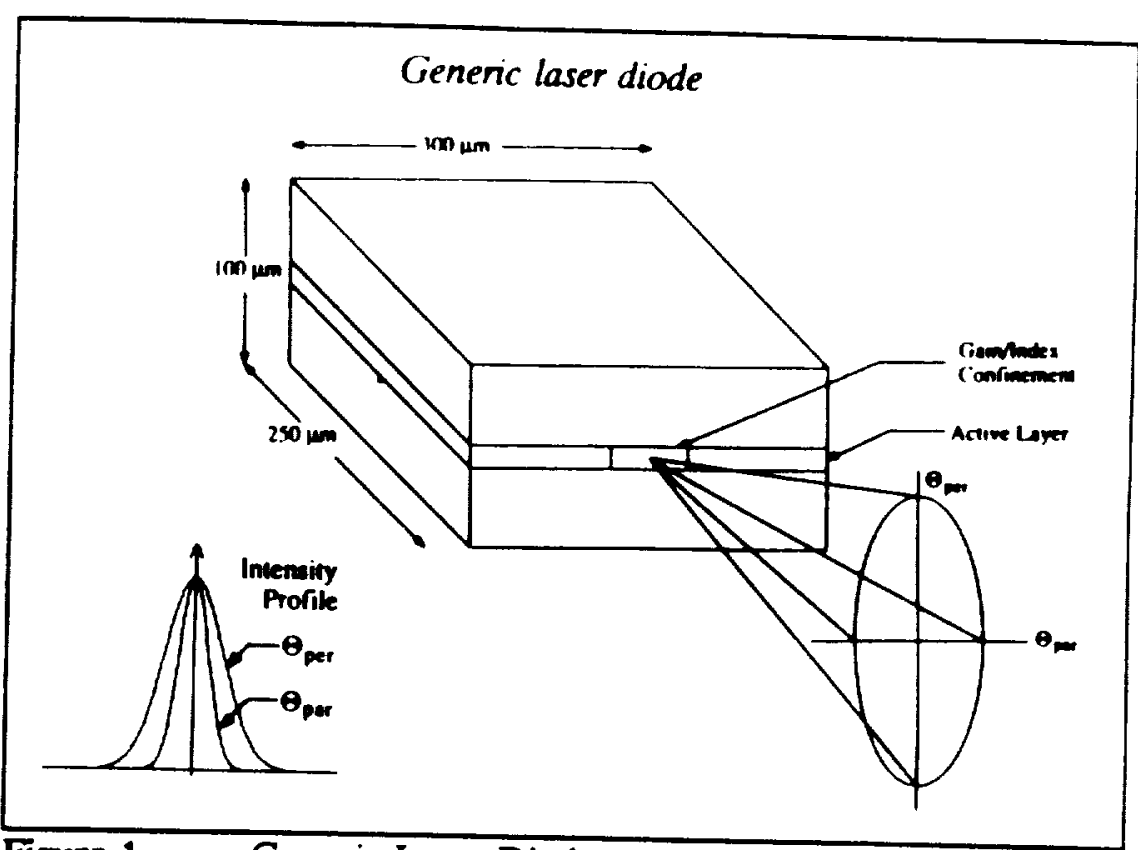

Figure 1 Generic Laser Diode

\section{Modeline the Optical System}

The laser beam propagates from the diode and eventually is incident on the entrance pupil of the optical system. A geometrical optics analysis of the system is a valid method of characterizing the optical system. Ray-trace calculations may be performed in order to quantify aberrations in the system. Figure 2 shows a portion of a generic M-O optical data storage system. The light from the diode is collimated, circularized, focused down to a spot on the disk, and the reflected light containing information is delivered to data and servo detectors. The following paragraphs describe a method of modeling the entrance pupil to exit pupil mapping of the optical system.

The wavefront is propagated to the entrance pupil of the system with scalar diffraction. The optical system maps the wavefront incident on the entrance pupil to the exit pupil of the system. Then, the wavefront is propagated to the disk with scalar diffraction. This modeling of the pupil to pupil mapping is accomplished in a two pronged approach as shown in Figure 3.

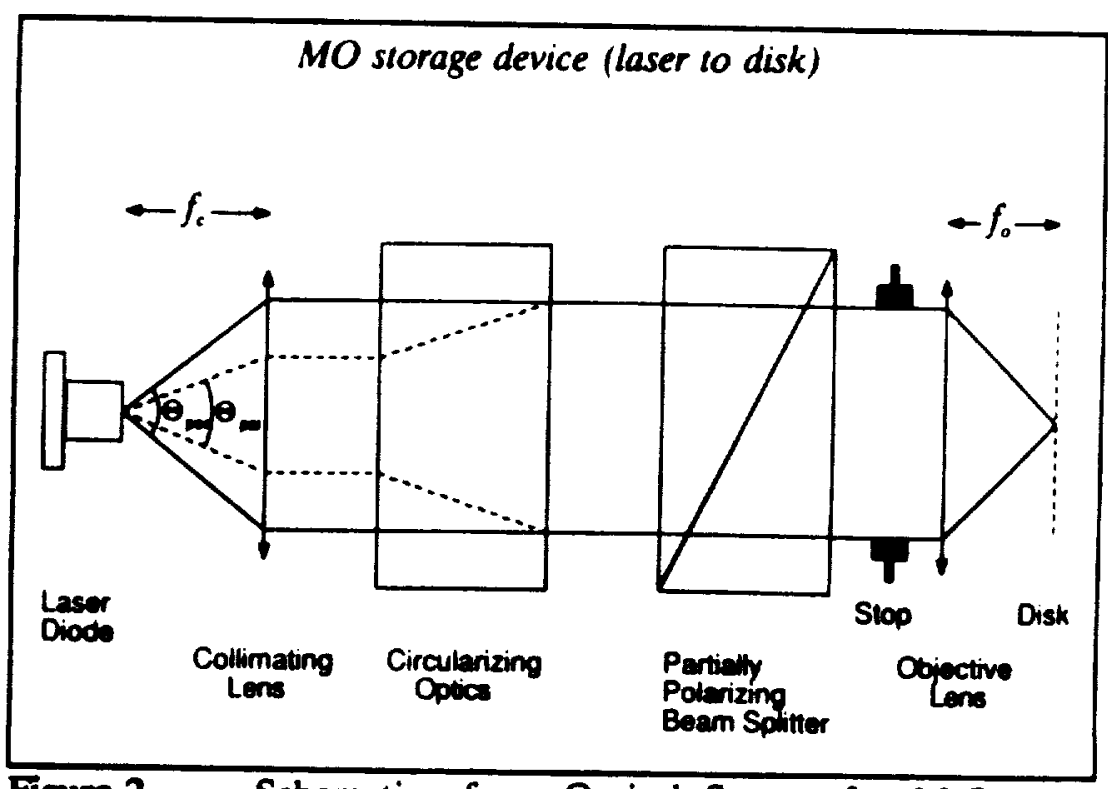

Figure 2 Schematic of an Optical System for M-O Data Storage 
To begin, we compute the ideal diffraction mapping of the entrance pupil to the exit pupil. We calculate a paraxial system quantifier. the $A B C D$ matrix. An $A B C D$ matrix is developed for all the elements in the system. and the resulting system $A B C D$ matrix is simply the product of all component matrices:

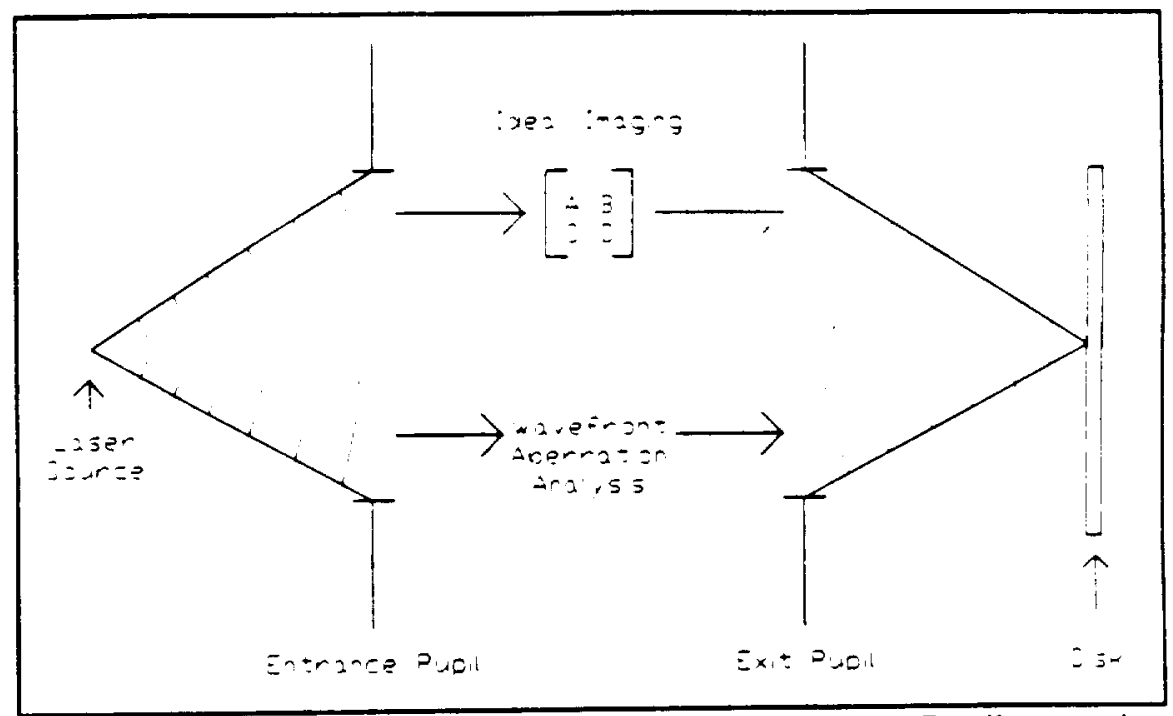

Figure 3 Diagram of Entrance Pupil to Exit Pupil mapping technique

$$
A B C D_{\text {sorem }}=\prod_{n=1}^{1} A B C D_{n}
$$

In the above equation, the optical system has $\mathrm{i}$ elements. Once the system $A B C D$ matrix has been found, that information is placed into the Huygens' integral for wave propagation (8). In the following equation, $x_{1}=$ entrance pupil plane, $x_{2}=$ exit pupil plane, and $\bar{u}_{i}\left(x_{1}\right)$ is the wavefront at the given plane. Computation of the integral will map the wavefront from entrance to exit pupil, that is,

$$
\tilde{u}_{2}\left(x_{2}\right)=e^{-j k L} \cdot \iint \tilde{K}\left(x_{2}, x_{1}\right) \tilde{u}_{1}\left(x_{1}\right) d x_{1}
$$

The Huygens' kernel in one transverse dimension is given by:

$$
\tilde{K}\left(x_{2}, x_{1}\right)=\sqrt{\frac{j}{B \lambda_{0}}} \exp \left[-j \frac{\pi}{B \lambda_{0}}\left(A x_{1}^{2}-2 x_{1} x_{2}+D x_{2}^{2}\right)\right] .
$$

The aberrations in the optical system must now be considered. To compute the aberrations, a ray-trace of the system is performed. An ideal lens may be placed at the exit pupil, and that lens will focus the rays to an image plane. At the arbitrary image plane, the two-dimensional transverse ray fan is calculated. If that ray fan is integrated, the result is the optical path difference (OPD) in the exit pupil due to the aberrations in the system.

The ideal diffraction imaging wavefront and the OPD in the exit pupil due to aberrations are combined to give the total exit pupil wavefront. This is simply the addition of the two component phase fronts. The OPD due to aberrations, plus the ideal diffraction phase front equals the exit pupil phase front. The amplitude of the beam at the exit pupil is given by the ideal diffraction propagation of the amplitude of the E-field incident on the entrance pupil. 
The wavefront that is now at the exit pupil is propagated to the optical disk using scalar diffraction calculations. The light interacts with the disk, and it is reflected from the disk back into the optical system. The reflected light propagates to detectors where the data is converted into an electrical signal.

\section{Modeling Disk Interaction}

Jones calculus is utilized in the analysis of the disk-laser beam interaction (2). The following column vector represents an arbitrary E-field, where $E_{\mathrm{x}}$ and $\mathrm{E}_{\mathrm{\gamma}}$ symbolize two orthogonal polarization components:

$$
E=\left(\begin{array}{l}
E_{x} \\
E_{y}
\end{array}\right)
$$

$E_{\mathrm{x}}$ and $\mathrm{E}_{\mathrm{\gamma}}$ are the matrices of complex values that model the wavefront. The light that is incident on the disk is linearly polarized, and the direction of polarization is defined as the $x$ direction. The following column vector represents the incident light:

$$
E_{l}=\left(\begin{array}{c}
E_{x} \\
0
\end{array}\right)
$$

This light interacts with the disk. The disk may be described using the following Jones matrix:

$$
D=\bar{F}\left(\begin{array}{cc}
1 & k e^{i 8} \\
k e^{i 8} & -1
\end{array}\right),
$$

where $\vec{t}$ is the bulk reflectivity of the medium, $\mathrm{K}$ is a matrix which contains information about the tangent of the Kerr angle at sampled positions on the disk, and 8 is the phase shift between the $x$ and y components on reflection from the medium (2). The reflected light is the product of the disk matrix and the incident light:

$$
E_{r}=D \cdot E_{i}=\bar{r}\left(\begin{array}{cc}
1 & k e^{i s} \\
k e^{i b} & -1
\end{array}\right)\left(\begin{array}{c}
E_{x} \\
0
\end{array}\right)=\bar{r}\left(\begin{array}{c}
E_{x} \\
k E_{x} e^{i b}
\end{array}\right)
$$

$K$ contains the information about the data marks on the disk. Marks, which correspond to logical ones have a value equal to $+\tan (\mathrm{Kerr}$ angle). Spaces between the marks, which correspond to logical zeros, have a value equal to $-\tan$ (Kerr angle). Figure 4 graphically depicts the matrix elements in a sample $x$ matrix.

The two polarization components in the reflected field are separately propagated back through the optical system using of Fresnel diffraction calculations. The methods described in the sections above are used to model propagation of the reflected light back through the system to data and servo detectors, which convert the information contained in the reflected beam into an electrical signal. 


\section{Implementation}

This model is implemented on a 386 computer, running at $25 \mathrm{MHz}$. The modeling environment is the MATLAB programming language. MATLAB is an interactive mathematics programming environment that allows a user to perform a variety of calculations, including those necessary for the scalar diffraction model of the light propagating through the system.

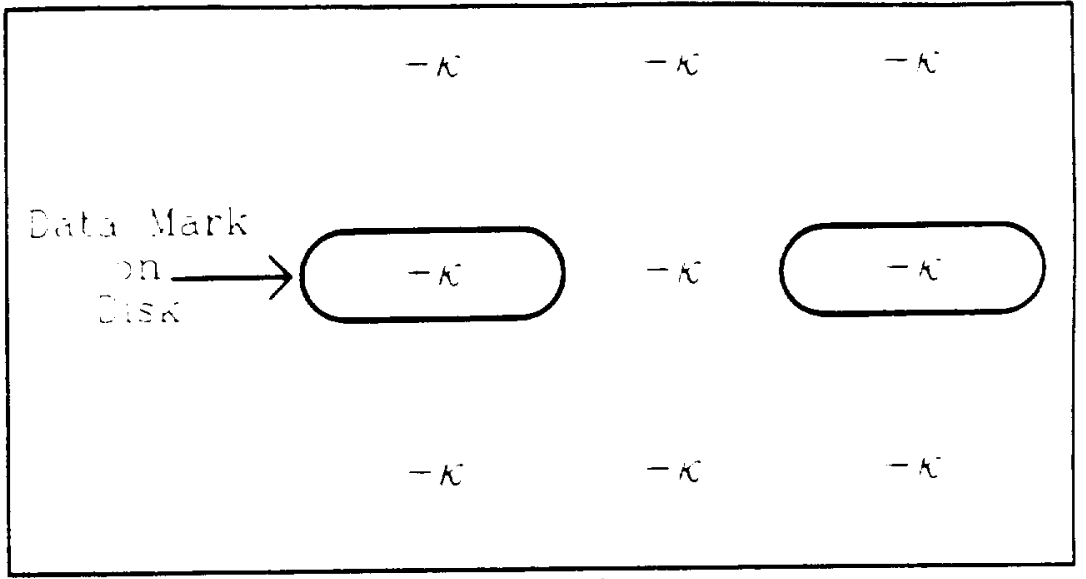

Figure $4 \quad$ Example of a $k$ matrix

\section{Conclusion}

Light that travels through an optical data storage system can be modeled using the techniques discussed above. We can treat the modeling of light differently according to the functional subsystems interacting with the light. As discussed above, these subsystems are the laser diode, the optical system, and the optical disk. The model of the laser beam in the system is a combined physical optics analysis and geometrical optics analysis. The model uses Fresnel diffraction calculations and ray-trace calculations in order to simulate the propagation of the laser beam through the system.

\section{Acknowledgements}

The authors wish to thank Ken Moore of the Optical Sciences Center for suggesting the combination of ray-trace and diffraction calculations necessary to find the exit pupil wavefront. This work was supported by NASA grant no. NGT-50579.

\section{References}

1. M. Mansuripur, "Certain computational aspects of vector diffraction problems", JOSA $A$ v. 6 , no. 5 (1989)

2. T. Milster, T. Gardner, "Modeling of a magneto-optic read path", Proc. SPIE v. 899 (1988)

3. C. Perlov, H. Birecki, "Modeling the magneto-optic recording process", JJAP v. 28 (1989)

4. A.B. Marchant, Optical recording: a technical overview, Addison-Wesley Inc. (1990)

5. J.W. Goodman, Introduction to Fourier Optics, McGraw-Hill Inc. (1968)

6. W.P. Dumke, The angular beam divergence in double-heterojunction lasers with very thin active regions", IEEE Joumal of Quantum Electronics v. QE-11, no. 7 (1975)

7. D.D. Cook, F.R. Nash, "Gain-induced guiding and astigmatic output of GaAs lasers", Journal of Applied Physics v. 46, no. 4 (1975)

8. A.E. Siegman, Lasers, University Science Books (1986) 
Appendix I 